\title{
A robust approach for analyzing unbalanced factorial designs with fixed levels
}

\author{
Guillermo VALLEJo \\ University of Oviedo, Oviedo, Spain \\ Manuel Ato \\ University of Murcia, Murcia, Spain \\ AND \\ M. Paula Fernández \\ University of Oviedo, Oviedo, Spain
}

\begin{abstract}
The goal of this study was to investigate the performance of Hall's transformation of the Brunner-DetteMunk (BDM) and Welch-James (WJ) test statistics and Box-Cox's data transformation in factorial designs when normality and variance homogeneity assumptions were violated separately and jointly. On the basis of unweighted marginal means, we performed a simulation study to explore the operating characteristics of the methods proposed for a variety of distributions with small sample sizes. Monte Carlo simulation results showed that when data were sampled from symmetric distributions, the error rates of the original BDM and WJ tests were scarcely affected by the lack of normality and homogeneity of variance. In contrast, when data were sampled from skewed distributions, the original BDM and WJ rates were not well controlled. Under such circumstances, the results clearly revealed that Hall's transformation of the BDM and WJ tests provided generally better control of Type I error rates than did the same tests based on Box-Cox's data transformation. Among all the methods considered in this study, we also found that Hall's transformation of the BDM test yielded the best control of Type I errors, although it was often less powerful than either of the WJ tests when both approaches reasonably controlled the error rates.
\end{abstract}

Factorial designs are used successfully for a wide range of psychological applications. In fact, a review of the two major Spanish psychological journals (Psicothema and International Journal of Clinical and Health Psychology) revealed that of the 952 articles published between 2000 and 2005 , almost $20 \%$ used some form of factorial design. These designs offer certain advantages over conventional comparative designs. The major advantage is that they allow researchers to investigate the influence of two or more variables acting and interacting simultaneously. Another advantage is that they provide more information by using only a fraction of the subjects that would be required if separate experiments were conducted. They also enhance the experimental control and increase the generalizability of results.

As is well known and well documented, over the last 4 decades, a debate has emerged regarding which is the best solution for the analysis of unbalanced factorial designs. Researchers having opted for least-squares solutions performed by comparing models (i.e., comparing a full model that contains all effects with a restricted model that contains all effects except the effect of interest; basically, the model comparison approach works by placing constraints on the parameters of a full model to obtain a more parsimonious model of the data), a great part of the debate has focused on deciding on the most adequate model for testing the hypotheses of interest (for a fuller review, see MacNaughton, 1998, and the references therein). For example, in a two-factor ANOVA model with an unequal number of observations in each treatment combination (cell), one can obtain at least three different decompositions of the sum of squares (SS) - namely, Types I, II, and III SS (the SAS and SPSS statistical packages use these three designators). Although the preferred method of analysis is still the source of some controversy (Maxwell \& Delaney, 2004), most of the analyses are performed by equally weighting the marginal means (i.e., on the basis of SAS Type III SS). Nevertheless, it is crucial to bear in mind that the choice of the appropriate method depends on the question the experimenter wants to answer about the data.

When the effects of the factorial design are tested using ordinary least squares estimation based on the model comparison approach, it is assumed that the random errors are independently normally distributed with zero mean and common variance. It is important to note that several authors, including Lee and Ahn (2003), have shown that the

G.Vallejo,gvallejo@uniovi.es 
usual $F$ test is too liberal (conservative) when the sample sizes are negatively (positively) correlated with the variances. This problem can be critical in the case of a severe degree of cell size imbalance, particularly when the sample sizes are small. As a consequence, some researchers have been reluctant to use the conventional ANOVA model for the analysis of unbalanced factorial designs. Fortunately, there are several options that can be used for overcoming the negative impact of the heterogeneity of variances on rates of Type I error. However, both the computational effort and the quality of the inference reveal important differences between them (for the one-way ANOVA, see, e.g., the work of Krishnamoorthy, Lu, \& Mathew [2007], Kulinskaya \& Dollinger [2007], and the references cited therein).

For heteroscedastic factorial designs with small to moderate sample sizes, Keselman, Carriere, and Lix (1995) demonstrated that a simple Welch-James (WJ hereafter) type statistic developed by Johansen (1980) performed well with respect to their Type I error rate and power when the data were normally distributed. When the data were skewed, the WJ approach required an increase of cell sizes in order to control the error rate. Subsequently, Keselman, Kowalchuk, and Lix (1998) proposed using robust location and scale estimators (e.g., trimmed means and Winsorized variances), rather than the usual least squares estimators, to reduce the biasing effects of nonnormality. Moreover, Luh and Guo (2004) reported that the WJ test improved its performance when both Johnson's (1978) and Hall's (1992) transformation methods were applied in conjunction with robust estimators. However, in addition to the importance of having a minimum number of subjects per cell when the data are trimmed, this method alters the hypotheses that are tested. When distributions are asymmetric, hypotheses about trimmed means are not the same as hypotheses about means. Nevertheless, it is also worth noting that means can be misleading because they do not represent the center of the data, whereas trimmed means do a better job at representing the center of the data.

Another simple method that can also be used for testing hypotheses in factorial designs under a normal heteroscedastic error structure is the generalization of Box's (1954) method proposed by Brunner, Dette, and Munk (1997; BDM hereafter). Recently, Richter and Payton (2003) assessed the operating characteristics of the BDM test, assuming normally distributed responses. Their results highlighted the fact that this approach limited the number of errors to the nominal value when the variances were unequal and the cell sizes were relatively small. Most recently, Vallejo, Fernández, and Livacic-Rojas (2010) investigated the joint impact of violating variance homogeneity and normality on the performance of BDM and WJ procedures in analyzing parametric factorial designs. In their numerical study, the authors found that when the shape of the distribution was symmetric, both approaches consistently controlled the rates of error. However, Vallejo et al. (2010) also demonstrated that when data were obtained from the exponential and lognormal distributions, there were serious mistakes of inference with these methods; that is, their Type I error control failed. This pattern of results was consistent across the three SSs-Type I,
Type II, and Type III-employed to test the hypotheses. For this reason, it was imperative that the researchers chose statistical techniques that were robust to the violations of the distributional assumptions.

The most commonly used approach for improving the normality of variables is to transform the scale of the data by nonlinear functions. When researchers know the theoretical distribution of the data, they can utilize this information in selecting a transformation. For example, if the data tend to follow a Poisson distribution, the square root transformation will be used. When prior information about the data cannot be used to help in choosing an appropriate transformation, it is useful to estimate the power parameter using the method introduced by Box and Cox (1964), whereby it is assumed that the transformations can achieve simultaneously both normalization and homogeneity of variances. Once an appropriate transformation has been selected and the data analyzed on the new measurement scale, the interpretation of the results must be made on the selected scale.

A different approach for dealing with departures from normality, especially in terms of skewness and peakness, via symmetrizing transformations has been offered by Johnson (1978) and Hall (1992). As was noted previously, Luh and Guo (2004) found that Johnson's and Hall's transformation methods of the WJ test statistic, used in conjunction with robust estimators, improved its performance when it was used for comparing means in the twoway layout under extreme conditions of nonnormality and variance heterogeneity. Hall argued, and reported simulation evidence to demonstrate, that his empirical and general transformation is more efficient than that suggested by Johnson in cases of severely skewed distributions and small sample sizes. Also, Luh and Guo found Hall's invertible transformation to be slightly more powerful than Johnson's transformation method.

Accordingly, in this article, we employ a transformation based on Hall's (1992) work to contend with skewness and two heteroscedastic statistics (i.e., WJ and BDM) to contend with variance heterogeneity. Furthermore, for purposes of comparison, we shall study the performance of the WJ and BDM procedures with transformed data within the Box-Cox family. The advantage of this usual approach is that most statistical software packages (e.g., SAS, STATA, or S-PLUS/R) offer it as a standard feature. However, it does not always find a suitable transformation; it is available only for data that are positive, and the interpretation of the results has led to some controversy and debate. In all cases, the operating characteristics of the methods proposed (i.e., significance level and power) will be assessed by using a Type III SS ANOVA via Monte Carlo simulation.

\section{Definition of the Statistical Tests}

Consider a two-way layout with $j=1, \ldots, a$ levels of the factor $A, k=1, \ldots, b$ levels of the factor $B$, and $i=1, \ldots, n_{j k}$ scores for each of the $a b$ treatment combinations (cells). In terms of the full-rank (FR) or means model, the $i$ th observation from the $j k$ th cell of the factorial design is modeled by $Y_{i j k}=\mu_{j k}+e_{i j k}$, where $\mu_{j k}$ 
represents the population mean of the $j k$ th cell and $e_{i j k}$ are error terms that are assumed to be mutually independent and normally distributed with $E\left(e_{i j k}\right)=0$ and $\operatorname{Var}\left(e_{i j k}\right)=$ $\sigma^{2}$. Sometimes the responses are modeled by $Y_{i j k}=\mu+$ $\alpha_{j}+\beta_{k}+\gamma_{j k}+e_{i j k}$, where $\mu$ is the overall mean, $\alpha_{j}\left(\beta_{k}\right)$ is the effect of the $j$ th $\left(k\right.$ th) level of $A(B)$, and $\gamma_{j k}$ is the joint effect of treatment levels $j$ and $k$. The link between the FR model and the less full rank (LFR) model is $\mu_{j k}=$ $\mu+\alpha_{j}+\beta_{k}+\gamma_{j k}$, with the constraints $\Sigma_{j=1} \alpha_{j}=\Sigma_{k=1} \beta_{k}=$ $\Sigma_{j=1} \gamma_{j k}=\Sigma_{k=1} \gamma_{j k}=0$, since the LFR model is overparameterized (i.e., the number of cell means is less than the number of model parameters to be estimated).

From an FR model perspective, the general linear hypothesis can be written using matrix notation as follows:

$$
H_{0}(t): \mathbf{C}_{t} \boldsymbol{\mu}=\mathbf{0}, \quad(t=A, B, \text { or } A B),
$$

where $\mathbf{C}_{t}$ is the contrast matrix associated with the specific hypothesis, $\boldsymbol{\mu}$ is a vector column of population cell means, and $\mathbf{0}$ is the null vector. The contrast matrices $\mathbf{C}_{t}$ to test the hypotheses $H_{0}(A), H_{0}(B)$, and $H_{0}(A B)$ can be written as $\mathbf{C}_{A}=\mathbf{P}_{a} \otimes(1 / b) \mathbf{1}_{b}^{\prime}, \mathbf{C}_{B}=(1 / a) \mathbf{1}_{a}^{\prime} \otimes \mathbf{P}_{b}$, and $\mathbf{C}_{A B}=\mathbf{P}_{a} \otimes \mathbf{P}_{b}$. Here, $\mathbf{P}_{a}=\left(\mathbf{1}_{a-1} \mid-\mathbf{I}_{a-1}\right)$ and $\mathbf{P}_{b}=$ $\left(\mathbf{1}_{b-1} \mid-\mathbf{I}_{b-1}\right)$ are matrices of between-subjects contrasts with full-row rank, where $\mathbf{I}_{b}$ and $\mathbf{I}_{a}$ are identity matrices of dimensions $b$ and $a$, respectively, and the circle times operator $[\otimes]$ denotes the direct product of the matrices (this operator produces a matrix by multiplying each element of the first of the matrices by the whole other matrix). Similarly, the contrast vectors for conducting multiple comparisons among the marginal means and interaction contrasts are defined as $\mathbf{c}_{A}=\mathbf{p}_{j j^{\prime}} \otimes \mathbf{1}_{k}^{\prime}, \mathbf{c}_{B}=\mathbf{1}_{j}^{\prime} \otimes \mathbf{p}_{k k^{\prime}}$, and $\mathbf{c}_{A B}=\mathbf{p}_{j j^{\prime}} \otimes \mathbf{p}_{k k^{\prime}}$, where $\mathbf{p}_{j j^{\prime}}$ contains the coefficients that contrast the $j$ th and $j^{\prime}$ th row means and $\mathbf{p}_{k k^{\prime}}$ contains the coefficients that contrast the $k$ th and $k^{\prime}$ th column means.

The Welch-James Procedure. As was mentioned earlier, the usual ANOVA is very sensitive to departures from the homogeneity assumption, particularly in the unbalanced case. To overcome this problem, Welch (1951) and James (1951) suggested weighting the terms appearing in the $F$ ratio, to account for the different population variances, and estimating denominator degrees of freedom $(d f \mathrm{~s})$ from the data. According to Johansen (1980) and Keselman et al. (1995), the WJ type statistic is given by

$$
T_{\mathrm{WJ}}=\left(\mathbf{C}_{t} \hat{\boldsymbol{\mu}}\right)^{\prime}\left(\mathbf{C}_{t} \hat{\Sigma} \mathbf{C}_{t}^{\prime}\right)^{-1}\left(\mathbf{C}_{t} \hat{\boldsymbol{\mu}}\right),
$$

where $\mathbf{C}_{t}$ is as defined before, $\hat{\boldsymbol{\mu}}=\overline{\mathbf{Y}}=\left(\overline{\mathbf{Y}}_{1}^{\prime}, \ldots, \overline{\mathbf{Y}}_{a}^{\prime}\right)^{\prime}$, $\overline{\mathbf{Y}}_{j}=\left(\bar{Y}_{j 1}, \ldots, \bar{Y}_{j b}\right)^{\prime}, \bar{Y}_{j k}=\sum_{i=1}^{n_{j k}} Y_{i j k} / n_{j k}$, and $\hat{\Sigma}=\operatorname{diag}\left(\hat{\sigma}_{11}^{2} /\right.$ $\left.n_{11}, \ldots, \hat{\sigma}_{a b}^{2} / n_{a b}\right)$. Under $H_{0}, T_{\mathrm{WJ}} / c$ is distributed approximately as an $F$ variable with $\hat{v}_{1}=R\left(\mathbf{C}_{t}\right)$ and $\hat{v}_{2}=$ $\hat{v}_{1}\left(\hat{v}_{1}+2\right) / 3 M d f$, where $c=\hat{v}_{1}+2 M-(6 M) /\left(\hat{v}_{1}+2\right)$ and $R\left(\mathbf{C}_{t}\right)$ denotes the rank of $\mathbf{C}_{t}$. The formula for the statistic, $M$, is provided in Keselman et al. (1995).

The Brunner-Dette-Munk procedure. Brunner et al. (1997) proposed a modification of the quadratic forms used in the usual ANOVA with estimated $d f$ based on a classical moment-matching approach due to Box (1954). According to Brunner et al., the BDM test statistic is given by

$$
F_{\mathrm{BDM}}=N\left(\hat{\boldsymbol{\mu}}^{\prime} \mathbf{H}_{t} \hat{\boldsymbol{\mu}}\right) / \operatorname{tr}\left(\mathbf{H}_{t D} \hat{\mathbf{S}}\right),
$$

where $N=\Sigma_{j=1}^{a} \Sigma_{k=1}^{b} n_{j k}$ is the total sample size, $\mathbf{H}_{t}=\mathbf{C}_{t}^{\prime}$ $\left(\mathbf{C}_{t} \mathbf{D} \mathbf{C}_{t}^{\prime}\right)^{-1} \mathbf{C}_{t}$ is a matrix of range $\mathbf{C}_{t}, \mathbf{H}_{t D}$ denotes the diagonal matrix of the elements of $\mathbf{H}_{t}, \hat{\mathbf{S}}=N \mathbf{D} \hat{\boldsymbol{\Sigma}}$, D is a diagonal matrix with $1 / n_{j k}$ as its $j k$ th diagonal element, and $\operatorname{tr}(\cdot)$ denotes the trace of a square matrix (i.e., the sum of its diagonal elements). Under $H_{0}: \mathbf{C}_{t} \boldsymbol{\mu}=\mathbf{0}$ the authors proposed approximating the null distribution of $F_{\mathrm{BDM}}$ statistic by the central $F\left(\hat{v}_{1}, \hat{v}_{2}\right)$ distribution with estimated $d f$

$$
\hat{v}_{1}=\frac{\left[\operatorname{tr}\left(\mathbf{H}_{t D} \hat{\mathbf{S}}\right)\right]^{2}}{\operatorname{tr}\left(\mathbf{H}_{t} \hat{\mathbf{S}} \mathbf{H}_{t} \hat{\mathbf{S}}\right)} \quad \text { and } \quad \hat{v}_{2}=\frac{\left[\operatorname{tr}\left(\mathbf{H}_{t D} \hat{\mathbf{S}}\right)\right]^{2}}{\operatorname{tr}\left(\mathbf{H}_{t D}^{2} \hat{\mathbf{S}}^{2} \tilde{\mathbf{D}}\right)} \text {, }
$$

where $\tilde{\mathbf{D}}$ is a diagonal matrix with $1 /\left(n_{j k}-1\right)$ as its $j k$ th diagonal element. The error $d f \hat{v}_{2}$ is identical to the approximation provided by Vallejo and Ato (2006).

Finally, as pointed out by the same authors, the proposed approach can also be applied to the problem of testing hypotheses in nonparametric factorial designs, even if homogeneity is assumed for the $Y_{i j k} \mathrm{~s}$, because rank statistics are heteroscedastic in general (Akritas, 1990).

Hall's transformation of the BDM and WJ test statistics. To eliminate the effect of the skewness of an asymmetric statistic, Hall (1992) proposed the following transformation:

$$
T_{H}=T_{H}(S)=S+n^{-1} b \hat{\gamma}+a \hat{\gamma} S^{2}+\frac{1}{3} a^{2} \hat{\gamma}^{2} S^{3},
$$

where $S=(\hat{\theta}-\theta) / \hat{\sigma}, \hat{\theta}=\bar{Y}=n^{-1} \sum_{i=1}^{n} Y_{i}, \hat{\sigma}^{2}=$ $n^{-1} \sum_{i=1}^{n}\left(Y_{i}-\bar{Y}\right)^{2}, \hat{\gamma}$ is the skewness, defined by $\hat{\gamma}=$ $n^{-1} \sum_{i=1}^{n}\left(Y_{i}-\bar{Y}\right)^{3 /} \hat{\sigma}^{3}, a=1 / 3, b=1 / 6$, and $Y_{i}(i=1$, $2, \ldots, n)$ is a random independent sample from a single group.

To apply the method described in Hall's (1992) article to handle the problem of asymmetry in a two-way layout, replace the sample size $n$, sample mean $\hat{\theta}$, sample variance $\hat{\sigma}^{2}$, and sample skewness $\hat{\gamma}$ with $n_{j k},\left(\bar{Y}_{j k}-\bar{Y}\right)=\left(\hat{\alpha}_{j}+\right.$ $\left.\hat{\beta}_{k}+\hat{\gamma}_{j k}\right), \hat{\sigma}_{j k}^{2}$, and $n_{j k}^{-1} \Sigma_{i=1}^{n_{k}}\left(Y_{i j k}-\bar{Y}_{j k}\right)^{3 /} \hat{\sigma}_{j k}^{3}$. If we substitute these values into Equation 5, it follows that a mean statistic for the $j k$ th cell with Hall's transformation is given by the expression

$$
\begin{aligned}
\tilde{Y}_{j k}=\tilde{\sigma}_{j k}^{-1}\left[\left(\bar{Y}_{j k}-\bar{Y}\right)\right. & +\frac{\hat{Y}_{j k}}{6 \tilde{\sigma}_{j k}^{2}}+\frac{\hat{Y}_{j k}}{3 \tilde{\sigma}_{j k}^{4}}\left(\bar{Y}_{j k}-\bar{Y}\right)^{2} \\
& \left.+\frac{\hat{Y}_{j k}^{2}}{27 \tilde{\sigma}_{j k}^{8}}\left(\bar{Y}_{j k}-\bar{Y}\right)^{3}\right],
\end{aligned}
$$

where $\hat{Y}_{j k}=n_{j k}^{-2} \sum_{i=1}^{n_{j k}}\left(Y_{i j k}-\bar{Y}_{j k}\right)^{3}, \tilde{\sigma}_{j k}^{2}=\hat{\sigma}_{j k}^{2} / n_{j k}$, and $\bar{Y}=$ $\Sigma_{j=1}^{a} \Sigma_{k=1}^{b} \bar{Y}_{j k} / a b$.

Next, to compute the WJ and BDM procedures based on Hall's (1992) transformation, we replace $\hat{\mu}$ in Equations 2 and 3 with the estimate vector containing the cell means transformed by Hall's method, $\tilde{\boldsymbol{\mu}}=\left(\tilde{\mathbf{Y}}_{1}^{\prime}, \ldots, \tilde{\mathbf{Y}}_{a}^{\prime}\right)^{\prime}$, with $\tilde{\mathbf{Y}}_{j}=\left(\tilde{Y}_{j 1}, \ldots, \tilde{Y}_{j b}\right)^{\prime}$. The transformed heteroscedastic statistics then become

$$
T_{\mathrm{HWJ}}=\left(\mathbf{C}_{t} \tilde{\boldsymbol{\mu}}\right)^{\prime}\left(\mathbf{C}_{t} \hat{\boldsymbol{\Sigma}} \mathbf{C}_{t}^{\prime}\right)^{-1}\left(\mathbf{C}_{t} \tilde{\boldsymbol{\mu}}\right)
$$

and

$$
F_{\mathrm{HBDM}}=N\left(\tilde{\boldsymbol{\mu}}^{\prime} \mathbf{H}_{t} \tilde{\boldsymbol{\mu}}\right) / \operatorname{tr}\left(\mathbf{H}_{t D} \hat{\mathbf{S}}\right) .
$$


Again, under $H_{0}: \mathbf{C}_{t} \boldsymbol{\mu}=\mathbf{0}$, it is reasonable to approximate the null distribution of $T_{\mathrm{HWJ}}$ and $F_{\mathrm{HBDM}}$ tests by the central $F$ distribution with no change in $d f$ previously specified for statistics $T_{\mathrm{WJ}}$ and $F_{\mathrm{BDM}}$.

The Box-Cox method of power transformation. As was previously noted, the $\mathrm{WJ}$ and $\mathrm{BDM}$ approaches were also computed with transformed data. Unfortunately, the choice of the best transformation is not generally obvious. Therefore, instead of assuming that we know the transformation that reduces data variation and makes the data more normal, we estimate the transformation, by using maximum likelihood, within the Box-Cox family. Specifically, the transformed values were obtained as follows: $Y_{i j k}^{\prime}=\left[\left(Y_{i j k}+\delta\right)^{\lambda}-1\right] / \lambda$ for $\lambda \neq 0$ and $Y_{i j k}^{\prime}=$ $\log \left(Y_{i j k}+\delta\right)$ for $\lambda=0$, where $Y_{i j k}$ is the raw data, $Y_{i j k}^{\prime}$ is the transformed data, $\delta$ is an arbitrary constant chosen so that all scores are greater than 0 , and $\lambda$ is the transformation parameter. The appropriate power $\lambda$ was selected by maximizing the function

$$
\begin{aligned}
l(\lambda)= & -\frac{N}{2} \log \left[\frac{1}{2} \sum_{i=1}^{n} \sum_{j=1}^{a} \sum_{k=1}^{b}\left(Y_{i j k}^{\prime}-\bar{Y}^{\prime}\right)^{2}\right] \\
& +(\lambda-1) \sum_{i=1}^{n} \sum_{j=1}^{a} \sum_{k=1}^{b} \log \left(Y_{i j k}+\delta\right),
\end{aligned}
$$

where $\bar{Y}^{\prime}=\Sigma_{j=1}^{a} \Sigma_{k=1}^{b} \bar{Y}_{j k} / a b$. The transformed data were then substituted for the original data in the calculations of the $\mathrm{WJ}$ and $\mathrm{BDM}$ approaches.

\section{A Monte Carlo Study}

In order to evaluate the robustness and sensitivity of the proposed approaches, we carried out two simulation studies using 2 two-way ANOVA designs $(2 \times 5)$ and $(3 \times 5)$. We chose to investigate designs with five levels of factor $B$, because this study replicates and extends one made by Vallejo et al. (2010). The first study focused on comparing the robustness of the approaches when the normality and homogeneity assumptions did not hold. With this aim, the following four variables were manipulated.

1. Total sample size. Since the procedures may be affected by the sample size, the performance of the test statistics was investigated using two different global sample size conditions. In the two-way factorial design with $a=2$ and $b=5, N=50$ and $N=100$, whereas in the two-way factorial design with $a=3$ and $b=5, N=104$ and $N=208$.

2. Degree of sample size imbalance. For each sample size condition, both a moderate and a severe degree of cell size inequality were explored, as indexed by a coefficient of sample size variation $(C)$, where $C=(1 / \bar{n})\left[\sum_{j k}\left(n_{j k}-\bar{n}\right)^{2} /\right.$ $a b]^{1 / 2}, \bar{n}$ being the average size of the cells. For $N=50$ and $C=.25$, the unequal cell sizes were (1) $n_{1 k}=(3,3,5,5,6)$ and $n_{2 k}=(4,5,6,6,7)$, and for $N=50$ and $C=.50$, the unequal cell sizes were (2) $n_{1 k}=(3,3,3,6,6)$ and $n_{2 k}=$ $(3,3,5,7,11)$. When $N=100$, the sizes of these cells were doubled. Whereas for $N=104$ and $C=.25$, the unequal cell sizes were (1) $n_{1 k}=(4,4,5,6,7), n_{2 k}=(6,6,8,8,9)$, and $n_{3 k}=(7,7,8,9,10)$, and for $N=104$ and $C=.50$, the unequal cell sizes were (2) $n_{1 k}=(2,3,4,5,7), n_{2 k}=$
$(3,6,8,10,12)$, and $n_{3 k}=(4,6,9,12,13)$. When $N=208$, the sizes of these cells were doubled.

3. Relationship between cell size and unequal variance. Positive and negative pairings of unequal variances and cell sizes were investigated. A positive pairing implies that the treatment combination with the smallest size is associated with the smallest variance, whereas a negative pairing implies that the treatment combination with the smallest size is associated with the largest variance. The heterogeneous variances we consider here were obtained as follows: $\sigma_{j k}^{2}=[(1+j \times k) / 2]^{2}$.

4. Population distribution shape. Although the procedures examined are based on the normality assumption, when we work with real data, it is common to find that the skewness $\left(\gamma_{1}\right)$ and kurtosis $\left(\gamma_{2}\right)$ indices divert from zero (Micceri, 1989), which could lead us to an incorrect interpretation of the results obtained. Therefore, in order to investigate the possible effects of distribution shape on the robustness of the tests, we generated data, both normal and nonnormal in form, sampling from the $g$-and- $h$ family of distributions introduced by Tukey (1977) and extensively studied by Headrick, Kowalchuk, and Sheng (2008), Hoaglin (1985), and Martinez and Iglewicz (1984). Specifically, besides the normal distribution $(g=h=0$; $\gamma_{1}=\gamma_{2}=0$ ) we chose to investigate distributions where (1) $g=0$ and $h=.109$, a distribution with skew and kurtosis equal to that for a Laplace (or double exponential) distribution $\left(\gamma_{1}=0\right.$ and $\left.\gamma_{2}=3\right)$; (2) $g=.76$ and $h=$ -.098 , a distribution with skew and kurtosis equal to that for an exponential distribution $\left(\gamma_{1}=2\right.$ and $\left.\gamma_{2}=6\right)$; and (3) $g=1$ and $h=0$, a distribution with skew and kurtosis equal to that for a lognormal distribution $\left(\gamma_{1}=6.18\right.$ and $\left.\gamma_{2}=110.94\right)$. The $g$-and- $h$ distributions were obtained by transforming the standard normal variable $Z_{i j k}$, generated using the SAS (SAS Institute, 2008) RANNOR function, to $Z_{i j k}^{*}=g^{-1}\left[\exp \left(g Z_{i j k}\right)-1\right] \exp \left(h Z_{i j k}^{2} / 2\right)$, where $g$ and $h$ are real numbers controlling skewness and kurtosis, respectively. It should be noted that when $g=0$, the $g$-and- $h$ distribution reduces to $Z_{i j k}^{*}=Z_{i j k} \exp \left(h Z_{i j k}^{2} / 2\right)$ which is also known as the $h$-distribution. Similarly, when $h=0$, the $g$-and- $h$ family reduces to $Z_{i j k}^{*}=\left[\exp \left(g Z_{i j k}\right)-1\right] / g$, which is also known as the $g$-distribution. Finally, to obtain a distribution with standard deviation $\sigma_{j k}$, each score on the dependent measure was created by using the linear model $Y_{i j k}=\mu_{j k}+\sigma_{j k} \times\left(Z_{i j k}^{*}-\mu_{g h}\right)$, where the population mean of the $g$-and- $h$ distribution is $\mu_{g h}=$ $\left\{\exp \left[g^{2} /(2-2 h)\right]-1\right\} /\left[g(1-h)^{1 / 2}\right]$.

The second study focused on the comparison of the sensitivity of the approaches for detecting the effects of the two-way factorial design with $a=3$ and $b=5$. Needless to say, the power analysis was performed only under those conditions in which the procedures reasonably controlled the error rates. With the aim of avoiding the occurrence of possible ceiling effects, we selected $\mu$ values that provide nominal powers $(1-\beta)$ of 0.60 . The theoretical powers were calculated using the general linear model as implemented through the SAS PROC GLM (SAS Institute, 2008) module when the normality and homogeneity of variance assumptions are satisfied. Employing the FINV function of the SAS, we discovered the values of the pa- 
rameters of noncentrality $\left(\lambda_{j}\right)$ so that $\operatorname{Prob}\left[F\left(v_{1}, v_{2}, \lambda_{j}\right)>\right.$ $\left.F_{.05}\left(v_{1}, v_{2}, 0\right)\right]=1-\beta$.

When our interest focused on investigating the sensitivity of the tests for detecting the real effect of the rows (variable $A$ ), the cells corresponding to the intersection of $a_{2} \cap b_{1}$ and $a_{2} \cap b_{5}$ took on the value $-c$ $\left(a_{2} b_{1}=a_{2} b_{5}=-c\right)$, and that corresponding to the intersection of $a_{3} \cap b_{1}$ and $a_{3} \cap b_{5}$ the value $c$. The remaining cells were kept unchanged throughout the study. On the other hand, when our interest was centered on calculating the power of the columns (variable $B$ ), the cells corresponding to the intersection of the levels $a_{2} \cap b_{1}$ and $a_{3} \cap b_{1}$ were set equal to $-c$, and those corresponding to the intersection of the levels $a_{2} \cap b_{5}$ and $a_{3} \cap b_{5}$ were set equal to $c$. Finally, when our interest resided in estimating the power of the $A B$ interaction, the cells referring to the intersection of the levels $a_{2} \cap b_{1}$ and $a_{3} \cap b_{5}$ took on the value $c$, and the cells referring to the intersection of the levels $a_{2} \cap b_{5}$ and $a_{3} \cap b_{1}$ the value $-c$. In all cases, the largest means were paired with the smallest variances.

Consistent with previous research (Brunner et al., 1997; Keselman et al., 1995; Luh \& Guo, 2004; Vallejo et al., 2010), 5,000 samples in each condition were created.
From the simulations, the observed proportions of calculated $F$ values falling beyond the appropriate .05 nominal level of significance were computed. Also, the empirical power rates based on the $F$ test were calculated as the number of data sets resulting in the rejection of $H_{0}$ divided by the total number of simulations. An SAS/IML macro was used for all calculations.

\section{Results of the Robustness Study}

We used Bradley's (1978) liberal criterion to facilitate the comparison between our results and those obtained by other researchers in similar studies. According to this criterion, those tests whose empirical error rate $\hat{\alpha}$ lies in the interval $0.5 \alpha \leq \hat{\alpha} \leq 1.5 \alpha$ would be considered robust. Therefore, for the nominal significance level employed in this study $(\alpha=5 \%)$ the interval used for defining the robustness of the tests was $2.5 \leq \hat{\alpha} \leq 7.5$. Correspondingly, a test procedure was considered too liberal (conservative) if its estimated Type I error rate was greater (smaller) than 7.5 (2.5).

The observed percentages for each set of 5,000 test statistics falling beyond the appropriate .05 critical value are presented in Tables $1-4$. The results are described jointly

Table 1

Empirical Type I Error Rates (in Percentages) for the $2 \times 5$ Factorial Design $(C=.25)$

\begin{tabular}{|c|c|c|c|c|c|c|c|c|c|c|c|c|c|}
\hline \multirow[b]{2}{*}{$N$} & \multirow[b]{2}{*}{$\mathrm{R}(N / V)$} & \multicolumn{3}{|c|}{$\begin{array}{c}\text { Distribution } \\
g=.000, h=.000\end{array}$} & \multicolumn{3}{|c|}{$\begin{array}{c}\text { Distribution } \\
g=.000, h=.109\end{array}$} & \multicolumn{3}{|c|}{$\begin{array}{c}\text { Distribution } \\
g=.76, h=-.098\end{array}$} & \multicolumn{3}{|c|}{$\begin{array}{c}\text { Distribution } \\
g=1.00, h=.000\end{array}$} \\
\hline & & $\mathrm{H}_{\mathrm{R}}$ & $\mathrm{H}_{\mathrm{C}}$ & $\mathrm{H}_{\mathrm{I}}$ & $\mathrm{H}_{\mathrm{R}}$ & $\mathrm{H}_{\mathrm{C}}$ & $\mathrm{H}_{\mathrm{I}}$ & $\mathrm{H}_{\mathrm{R}}$ & $\mathrm{H}_{\mathrm{C}}$ & $\mathrm{H}_{\mathrm{I}}$ & $\mathrm{H}_{\mathrm{R}}$ & $\mathrm{H}_{\mathrm{C}}$ & $\mathrm{H}_{\mathrm{I}}$ \\
\hline \multicolumn{14}{|c|}{ S-BDM } \\
\hline 50 & + & 4.53 & 4.23 & 3.99 & 4.65 & 3.47 & 3.46 & 4.17 & 2.58 & 2.80 & 3.60 & 1.77 & 1.65 \\
\hline 50 & - & 4.86 & 4.57 & 4.16 & 4.07 & 3.55 & 3.91 & 3.82 & 3.84 & 2.80 & 3.65 & 3.87 & 1.62 \\
\hline 100 & + & 4.56 & 4.71 & 4.52 & 4.97 & 4.31 & 4.25 & 4.82 & 3.77 & 3.73 & 4.76 & 2.33 & 2.30 \\
\hline 100 & - & 5.05 & 4.87 & 4.95 & 4.80 & 4.32 & 4.33 & 4.60 & 4.79 & 3.79 & 4.31 & 4.29 & 2.04 \\
\hline \multicolumn{14}{|c|}{$\mathrm{H}-\mathrm{BDM}$} \\
\hline 50 & + & 5.43 & 5.52 & 5.35 & 6.41 & 6.49 & 6.59 & 5.43 & 4.14 & 4.07 & 5.47 & 3.92 & 3.70 \\
\hline 50 & - & 5.96 & 5.99 & 6.00 & 5.94 & 5.60 & 5.99 & 5.27 & 5.55 & 4.47 & 5.99 & 6.39 & 4.40 \\
\hline 100 & + & 4.89 & 5.27 & 4.94 & 6.00 & 5.58 & 5.96 & 4.63 & 3.85 & 3.96 & 5.60 & 3.68 & 3.65 \\
\hline 100 & - & 5.39 & 5.60 & 5.49 & 5.74 & 6.41 & 5.80 & 4.41 & 4.21 & 3.38 & 5.07 & 4.75 & 3.09 \\
\hline \multicolumn{14}{|c|}{ BC-BDM } \\
\hline 50 & + & 4.17 & 4.30 & 4.07 & 4.25 & 3.58 & 3.80 & 5.97 & 4.59 & 4.26 & 6.91 & 4.83 & 3.23 \\
\hline 50 & - & 4.45 & 4.49 & 3.96 & 3.78 & 3.47 & 3.85 & 5.18 & 4.59 & 3.62 & 6.16 & 5.83 & 2.92 \\
\hline 100 & + & 3.67 & 4.54 & 4.31 & 4.10 & 4.17 & 4.36 & 7.45 & 6.12 & 4.73 & 10.12 & 7.51 & 4.75 \\
\hline 100 & - & 4.16 & 4.37 & 4.84 & 4.05 & 4.03 & 4.37 & 6.59 & 6.60 & 4.93 & 8.49 & 8.50 & 4.03 \\
\hline \multicolumn{14}{|l|}{ S-WJ } \\
\hline 50 & + & 4.53 & 4.26 & 4.47 & 4.65 & 3.40 & 3.49 & 4.17 & 5.98 & 3.03 & 3.60 & 6.73 & 2.02 \\
\hline 50 & - & 4.86 & 4.81 & 4.60 & 4.07 & 3.79 & 3.67 & 3.82 & 9.12 & 3.11 & 3.65 & 12.89 & 2.71 \\
\hline 100 & + & 4.56 & 4.97 & 4.62 & 5.20 & 4.57 & 4.31 & 4.82 & 6.49 & 4.10 & 4.76 & 7.68 & 3.03 \\
\hline 100 & - & 5.05 & 4.92 & 5.06 & 4.80 & 4.29 & 4.45 & 4.60 & 9.08 & 3.95 & 4.31 & 13.22 & 3.13 \\
\hline \multicolumn{14}{|l|}{$\mathrm{H}-\mathrm{WJ}$} \\
\hline 50 & + & 5.43 & 6.22 & 6.48 & 6.51 & 6.65 & 6.35 & 5.43 & 6.98 & 4.92 & 5.47 & 9.72 & 6.90 \\
\hline 50 & - & 5.96 & 6.43 & 6.19 & 5.91 & 6.22 & 5.90 & 5.27 & 9.55 & 5.54 & 5.99 & 14.64 & 6.96 \\
\hline 100 & + & 4.89 & 5.35 & 5.42 & 5.97 & 6.66 & 6.33 & 4.63 & 4.72 & 2.97 & 5.60 & 5.66 & 2.62 \\
\hline 100 & - & 5.39 & 5.90 & 6.18 & 6.03 & 6.37 & 6.06 & 4.41 & 6.73 & 2.82 & 5.07 & 9.91 & 2.22 \\
\hline \multicolumn{14}{|c|}{ BC-WJ } \\
\hline 50 & + & 4.17 & 4.19 & 4.52 & 4.25 & 3.44 & 3.52 & 5.97 & 6.99 & 4.86 & 6.91 & 8.73 & 3.29 \\
\hline 50 & - & 4.45 & 4.85 & 4.58 & 3.78 & 3.96 & 3.85 & 5.18 & 9.87 & 3.22 & 6.16 & 14.11 & 3.77 \\
\hline 100 & + & 3.67 & 4.61 & 4.50 & 4.10 & 4.04 & 4.36 & 7.45 & 7.87 & 5.03 & 10.12 & 11.14 & 5.05 \\
\hline 100 & - & 4.16 & 4.66 & 5.04 & 4.05 & 4.49 & 4.40 & 6.59 & 10.42 & 5.00 & 8.49 & 16.31 & 4.03 \\
\hline
\end{tabular}

Note- $g=(\cdot), h=(\cdot)$ specifies a particular $g$-and- $h$ distribution with specific values of skewness and kurtosis; $C$, coefficient of cell size variation; $N$, total sample size; $\mathrm{R}(N / V)$, relationship between the size of the cells and the size of the variances; $\mathrm{H}_{\mathrm{R}}$, hypothesis rows; $\mathrm{H}_{\mathrm{C}}$, hypothesis columns; $\mathrm{H}_{\mathrm{I}}$, hypothesis interaction; $\mathrm{S}-\mathrm{BDM}$, standard Brunner-Dette-Munk test; $\mathrm{H}-\mathrm{BDM}$, Hall's transformation BDM test; BC-BDM, BDM test based on Box-Cox's power transformation; S-WJ, standard WelchJames test; H-WJ, Hall's transformation WJ test; BC-WJ, WJ test based on Box-Cox's power transformation. Bold values are outside the [2.50-7.50] interval, for the $5 \%$ level of significance used in this study. 
Table 2

Empirical Type I Error Rates (in Percentages) for the $2 \times 5$ Factorial Design $(C=.50)$

\begin{tabular}{|c|c|c|c|c|c|c|c|c|c|c|c|c|c|}
\hline \multirow[b]{2}{*}{$N$} & \multirow[b]{2}{*}{$\mathrm{R}(N / V)$} & \multicolumn{3}{|c|}{$\begin{array}{c}\text { Distribution } \\
g=.000, h=.000\end{array}$} & \multicolumn{3}{|c|}{$\begin{array}{c}\text { Distribution } \\
g=.000, h=.109\end{array}$} & \multicolumn{3}{|c|}{$\begin{array}{c}\text { Distribution } \\
g=.76, h=-.098\end{array}$} & \multicolumn{3}{|c|}{$\begin{array}{c}\text { Distribution } \\
g=1.00, h=.000\end{array}$} \\
\hline & & $\mathrm{H}_{\mathrm{R}}$ & $\mathrm{H}_{\mathrm{C}}$ & $\mathrm{H}_{\mathrm{I}}$ & $\mathrm{H}_{\mathrm{R}}$ & $\mathrm{H}_{\mathrm{C}}$ & $\mathrm{H}_{\mathrm{I}}$ & $\mathrm{H}_{\mathrm{R}}$ & $\mathrm{H}_{\mathrm{C}}$ & $\mathrm{H}_{\mathrm{I}}$ & $\mathrm{H}_{\mathrm{R}}$ & $\mathrm{H}_{\mathrm{C}}$ & $\mathrm{H}_{\mathrm{I}}$ \\
\hline \multicolumn{14}{|c|}{ S-BDM } \\
\hline 50 & + & 4.78 & 3.92 & 4.43 & 4.13 & 4.29 & 3.86 & 3.87 & 2.45 & 2.15 & 3.52 & 1.73 & 1.46 \\
\hline 50 & - & 4.53 & 4.30 & 4.37 & 3.96 & 3.95 & 3.78 & 4.12 & 3.91 & 2.86 & 3.97 & 4.11 & 1.85 \\
\hline 100 & + & 5.06 & 4.35 & 4.36 & 4.77 & 4.85 & 4.15 & 4.61 & 3.91 & 2.86 & 4.58 & 2.27 & 1.94 \\
\hline 100 & - & 4.58 & 4.81 & 4.88 & 4.55 & 4.56 & 4.47 & 5.06 & 4.29 & 3.67 & 4.82 & 4.78 & 2.49 \\
\hline \multicolumn{14}{|c|}{$\mathrm{H}-\mathrm{BDM}$} \\
\hline 50 & + & 6.13 & 6.31 & 6.17 & 6.12 & 6.67 & 6.72 & 6.28 & 5.69 & 5.64 & 6.62 & 5.23 & 5.00 \\
\hline 50 & - & 6.13 & 6.00 & 6.12 & 5.64 & 5.98 & 5.64 & 6.43 & 6.83 & 6.21 & 6.65 & 7.64 & 6.48 \\
\hline 100 & + & 5.65 & 5.41 & 5.21 & 5.65 & 5.96 & 5.69 & 4.92 & 3.25 & 3.72 & 5.56 & 3.51 & 3.54 \\
\hline 100 & - & 5.24 & 5.53 & 5.86 & 6.43 & 6.15 & 6.98 & 4.14 & 3.85 & 3.75 & 5.19 & 4.88 & 3.49 \\
\hline \multicolumn{14}{|c|}{ BC-BDM } \\
\hline 50 & + & 4.48 & 3.79 & 3.97 & 4.05 & 3.48 & 3.65 & 5.34 & 3.89 & 3.60 & 6.81 & 3.29 & 2.55 \\
\hline 50 & - & 4.49 & 4.04 & 4.48 & 4.08 & 3.59 & 3.61 & 5.14 & 5.17 & 3.95 & 6.54 & 6.70 & 3.04 \\
\hline 100 & + & 4.64 & 4.06 & 4.33 & 4.30 & 3.95 & 4.00 & 6.93 & 5.20 & 4.11 & 9.98 & 6.44 & 3.98 \\
\hline 100 & - & 4.10 & 4.42 & 4.96 & 4.44 & 4.20 & 4.63 & 6.85 & 6.60 & 5.07 & 9.42 & 9.03 & 4.88 \\
\hline \multicolumn{14}{|l|}{ S-WJ } \\
\hline 50 & + & 4.78 & 4.07 & 4.43 & 4.13 & 4.08 & 3.54 & 3.87 & 5.18 & 2.77 & 3.52 & 4.89 & 1.79 \\
\hline 50 & - & 4.73 & 5.28 & 5.54 & 3.96 & 4.20 & 4.11 & 4.12 & 11.81 & 4.63 & 3.97 & 15.89 & 3.79 \\
\hline 100 & + & 5.26 & 4.72 & 4.66 & 4.77 & 4.24 & 4.36 & 4.61 & 5.95 & 3.58 & 4.58 & 6.46 & 2.25 \\
\hline 100 & - & 4.58 & 4.96 & 5.39 & 4.55 & 4.37 & 4.65 & 5.06 & 10.64 & 4.60 & 4.82 & 16.39 & 4.34 \\
\hline \multicolumn{14}{|l|}{$\mathrm{H}-\mathrm{WJ}$} \\
\hline 50 & + & 6.13 & 7.08 & 6.96 & 6.04 & 6.50 & 7.42 & 6.28 & 8.66 & 6.94 & 6.62 & 11.70 & 9.22 \\
\hline 50 & - & 6.13 & 7.40 & 7.79 & 5.07 & 5.82 & 7.88 & 6.43 & 13.70 & 9.03 & 6.90 & 19.13 & 11.23 \\
\hline 100 & + & 5.65 & 5.75 & 5.78 & 5.76 & 6.49 & 7.22 & 4.92 & 4.47 & 2.91 & 5.56 & 6.22 & 3.18 \\
\hline 100 & - & 5.24 & 6.06 & 6.37 & 6.39 & 6.07 & 7.79 & 4.14 & 7.94 & 3.68 & 5.19 & 12.98 & 4.22 \\
\hline \multicolumn{14}{|c|}{$\mathrm{BC}-\mathrm{WJ}$} \\
\hline 50 & + & 4.48 & 4.07 & 4.34 & 4.05 & 3.12 & 3.65 & 5.34 & 5.83 & 3.48 & 6.81 & 6.44 & 2.67 \\
\hline 50 & - & 4.49 & 5.45 & 5.85 & 4.08 & 4.52 & 4.31 & 5.14 & 11.90 & 5.85 & 6.54 & 16.81 & 5.65 \\
\hline 100 & + & 4.64 & 4.47 & 4.36 & 4.30 & 4.26 & 4.18 & 6.93 & 6.85 & 4.23 & 9.98 & 9.83 & 3.62 \\
\hline 100 & - & 4.10 & 4.91 & 5.37 & 4.44 & 4.02 & 4.78 & 6.85 & 11.82 & 6.49 & 9.42 & 19.11 & 6.49 \\
\hline
\end{tabular}

Note- $g=(\cdot), h=(\cdot)$ specifies a particular $g$-and- $h$ distribution with specific values of skewness and kurtosis; $C$, coefficient of cell size variation; $N$, total sample size; $\mathrm{R}(N / V)$, relationship between the size of the cells and the size of the variances; $\mathrm{H}_{\mathrm{R}}$, hypothesis rows; $\mathrm{H}_{\mathrm{C}}$, hypothesis columns; $\mathrm{H}_{\mathrm{I}}$, hypothesis interaction; $\mathrm{S}-\mathrm{BDM}$, standard Brunner-Dette-Munk test; $\mathrm{H}-\mathrm{BDM}$, Hall's transformation BDM test; BC-BDM, BDM test based on Box-Cox's power transformation; S-WJ, standard WelchJames test; H-WJ, Hall's transformation WJ test; BC-WJ, WJ test based on Box-Cox's power transformation. Bold values are outside the [2.50-7.50] interval, for the 5\% level of significance used in this study.

because the pattern of results was similar for two factorial designs. The simulation results indicate the following.

1. When sampling from a normal distribution, we found that all examined tests provided acceptable Type I error control, although Hall's (1992) transformation of the WJ test statistic became slightly liberal when $C=.50$, particularly when sample size was small and variances and cell sizes were negatively paired.

2. When sampling from a symmetric distribution with moderate degree of kurtosis (i.e., double-exponential-type data), descriptive analyses indicated that the original (i.e., BDM and WJ) and transformed BDM tests provided good Type I error control. Very similar results were obtained using the BDM test after applying a Box-Cox data transformation. On the other hand, it was also found that for the interaction, Hall's (1992) transformation of the WJ test was occasionally liberal when $C=.50$. The test presented no problems in relation to control of the error rates corresponding to the main effects.

3. When sampling from a distribution with moderate skew (i.e., exponential-type data), the only procedure that adequately controlled the error rates was Hall's (1992) transformation of the BDM test. The BDM and WJ ap- proaches failed to control Type I error, the former being occasionally conservative and the latter frequently liberal. The Box-Cox transformation had only a small effect on the error rates, relative to the untransformed data, but was occasionally liberal, particularly in the $3 \times 5$ design. With respect to the WJ procedure based on Hall's transformation method, the results shown in Tables $1-4$ indicate that the approach was frequently liberal but showed improved rates of Type I error control when cell size increased and the degree of cell size inequality decreased.

4. When sampling from a distribution with severe skew (i.e., lognormal-type data), no approach was able to control the error rates for all the studied conditions. The original BDM test was frequently conservative but showed substantially improved rates of Type I error control when used in conjunction with Hall's (1992) transformation method. Specifically, the original BDM was conservative for 21 of the 48 conditions studied, whereas it was liberal for 2 of the 48 conditions, applying Hall's transformation. On the other hand, the empirical error rates were frequently liberal for both the original WJ and transformed WJ test statistics. Furthermore, the use of the Box-Cox data transformation for testing the null hypotheses of the factorial 
Table 3

Empirical Type I Error Rates (in Percentages) for the $3 \times 5$ Factorial Design $(C=.25)$

\begin{tabular}{|c|c|c|c|c|c|c|c|c|c|c|c|c|c|}
\hline \multirow[b]{2}{*}{$N$} & \multirow[b]{2}{*}{$\mathrm{R}(N / V)$} & \multicolumn{3}{|c|}{$\begin{array}{c}\text { Distribution } \\
g=.000, h=.000\end{array}$} & \multicolumn{3}{|c|}{$\begin{array}{c}\text { Distribution } \\
g=.000, h=.109\end{array}$} & \multicolumn{3}{|c|}{$\begin{array}{c}\text { Distribution } \\
g=.76, h=-.098\end{array}$} & \multicolumn{3}{|c|}{$\begin{array}{c}\text { Distribution } \\
g=1.00, h=.000\end{array}$} \\
\hline & & $\mathrm{H}_{\mathrm{R}}$ & $\mathrm{H}_{\mathrm{C}}$ & $\mathrm{H}_{\mathrm{I}}$ & $\mathrm{H}_{\mathrm{R}}$ & $\mathrm{H}_{\mathrm{C}}$ & $\mathrm{H}_{\mathrm{I}}$ & $\mathrm{H}_{\mathrm{R}}$ & $\mathrm{H}_{\mathrm{C}}$ & $\mathrm{H}_{\mathrm{I}}$ & $\mathrm{H}_{\mathrm{R}}$ & $\mathrm{H}_{\mathrm{C}}$ & $\mathrm{H}_{\mathrm{I}}$ \\
\hline \multicolumn{14}{|c|}{ S-BDM } \\
\hline 104 & + & 5.02 & 4.81 & 4.40 & 4.40 & 3.68 & 3.74 & 4.30 & 3.86 & 2.85 & 3.57 & 2.51 & 1.28 \\
\hline 104 & - & 4.71 & 4.67 & 4.08 & 3.58 & 4.54 & 3.22 & 4.26 & 3.97 & 2.61 & 3.39 & 3.46 & 1.23 \\
\hline 208 & + & 5.00 & 4.79 & 5.06 & 4.28 & 4.86 & 5.02 & 4.73 & 4.36 & 3.42 & 4.28 & 3.22 & 1.88 \\
\hline 208 & - & 4.99 & 4.94 & 4.87 & 5.02 & 4.70 & 4.12 & 4.66 & 4.69 & 3.63 & 3.87 & 4.24 & 1.90 \\
\hline \multicolumn{14}{|c|}{ H-BDM } \\
\hline 104 & + & 5.73 & 5.50 & 5.49 & 6.42 & 6.52 & 6.65 & 4.46 & 4.04 & 3.67 & 5.03 & 4.15 & 3.00 \\
\hline 104 & - & 5.57 & 5.49 & 5.73 & 5.96 & 5.64 & 6.08 & 4.35 & 4.85 & 3.03 & 4.55 & 4.72 & 2.75 \\
\hline 208 & + & 5.19 & 5.07 & 5.66 & 5.96 & 5.56 & 5.95 & 4.91 & 4.60 & 3.68 & 5.82 & 4.99 & 4.16 \\
\hline 208 & - & 5.27 & 5.36 & 5.25 & 5.80 & 6.26 & 5.82 & 4.47 & 4.58 & 3.63 & 5.11 & 5.08 & 3.54 \\
\hline \multicolumn{14}{|c|}{ BC-BDM } \\
\hline 104 & + & 4.50 & 4.48 & 4.52 & 3.88 & 3.34 & 3.90 & 7.01 & 6.43 & 4.50 & 9.72 & 8.55 & 3.70 \\
\hline 104 & - & 4.24 & 4.39 & 4.16 & 3.32 & 4.24 & 3.78 & 6.66 & 6.00 & 4.13 & 8.38 & 8.26 & 3.17 \\
\hline 208 & + & 3.97 & 4.23 & 4.96 & 3.64 & 4.22 & 4.92 & 8.76 & 8.57 & 5.20 & 14.13 & 12.84 & 5.33 \\
\hline 208 & - & 4.28 & 4.26 & 4.79 & 4.18 & 4.17 & 4.31 & 8.05 & 8.49 & 5.15 & 12.69 & 13.16 & 5.06 \\
\hline \multicolumn{14}{|l|}{ S-WJ } \\
\hline 104 & + & 4.68 & 4.53 & 5.04 & 3.77 & 3.49 & 3.39 & 4.35 & 4.53 & 4.41 & 4.38 & 5.32 & 3.52 \\
\hline 104 & - & 4.60 & 4.67 & 5.44 & 4.38 & 3.76 & 5.42 & 4.66 & 9.73 & 8.43 & 4.41 & 14.51 & 7.86 \\
\hline 208 & + & 4.66 & 4.87 & 4.83 & 4.40 & 4.54 & 4.34 & 4.93 & 5.24 & 4.31 & 5.00 & 6.01 & 3.83 \\
\hline 208 & - & 4.45 & 4.80 & 5.05 & 4.20 & 4.75 & 4.76 & 5.46 & 8.89 & 6.59 & 5.13 & 13.42 & 6.47 \\
\hline \multicolumn{14}{|l|}{$\mathrm{H}-\mathrm{WJ}$} \\
\hline 104 & + & 5.63 & 5.84 & 6.94 & 6.23 & 6.90 & 6.81 & 5.47 & 6.05 & 6.31 & 6.66 & 7.73 & 8.08 \\
\hline 104 & - & 5.71 & 5.92 & 6.42 & 5.96 & 6.12 & 5.96 & 6.05 & 10.41 & 10.10 & 6.74 & 15.61 & 12.42 \\
\hline 208 & + & 5.19 & 5.63 & 6.00 & 6.12 & 6.78 & 7.48 & 5.10 & 4.74 & 3.69 & 6.39 & 6.43 & 3.69 \\
\hline 208 & - & 5.26 & 5.20 & 6.59 & 5.77 & 6.52 & 5.69 & 4.87 & 6.85 & 4.31 & 5.40 & 10.67 & 5.04 \\
\hline \multicolumn{14}{|c|}{$\mathrm{BC}-\mathrm{WJ}$} \\
\hline 104 & + & 4.20 & 4.25 & 4.96 & 3.43 & 3.31 & 3.23 & 6.41 & 5.62 & 4.87 & 8.69 & 7.91 & 4.52 \\
\hline 104 & - & 4.30 & 4.54 & 4.71 & 4.12 & 3.86 & 5.60 & 6.65 & 10.83 & 8.83 & 7.78 & 17.00 & 9.49 \\
\hline 208 & + & 4.02 & 4.38 & 4.67 & 4.34 & 3.92 & 4.28 & 8.86 & 7.99 & 5.04 & 13.83 & 11.59 & 5.55 \\
\hline 208 & - & 3.83 & 4.40 & 5.10 & 3.76 & 4.54 & 4.96 & 8.24 & 12.18 & 7.63 & 10.74 & 20.46 & 8.27 \\
\hline
\end{tabular}

Note- $g=(\cdot), h=(\cdot)$ specifies a particular $g$-and- $h$ distribution with specific values of skewness and kurtosis; $C$, coefficient of cell size variation; $N$, total sample size; $\mathrm{R}(N / V)$, relationship between the size of the cells and the size of the variances; $\mathrm{H}_{\mathrm{R}}$, hypothesis rows; $\mathrm{H}_{\mathrm{C}}$, hypothesis columns; $\mathrm{H}_{\mathrm{I}}$, hypothesis interaction; $\mathrm{S}-\mathrm{BDM}$, standard Brunner-Dette-Munk test; H-BDM, Hall's transformation BDM test; BC-BDM, BDM test based on Box-Cox's power transformation; S-WJ, standard Welch-James test; H-WJ, Hall's transformation WJ test; BC-WJ, WJ test based on Box-Cox's power transformation. Bold values are outside the $[2.50-7.50]$ interval, for the $5 \%$ level of significance used in this study.

design with the BDM and WJ approaches cannot be considered a robust alternative.

\section{Results of the Study of Power}

When the data were extracted from severely skewed distributions (i.e., lognormal-type data), no method adequately controlled the error rates, and when they were generated from moderately skewed distributions (i.e., exponential-type data), only Hall's (1992) transformation of the BDM test proved to be robust. Therefore, comparison of the sensitivity of the tests was limited to the data extracted from symmetric distributions with moderate degree of kurtosis (i.e., double-exponential-type data).

When our interest was centered on comparing the sensitivity of the standard BDM and WJ tests and their corresponding versions based on Hall's (1992) and Box-Cox's transformation methods for detecting the row, column, and interaction effects, our results indicate the following.

1. The original BDM test, Hall's (1992) transformation of the BDM test, and the BDM test based on Box-Cox's data transformation were slightly more powerful than the original WJ test, Hall's transformation of the WJ test, and the WJ test based on Box-Cox's data transformation for detecting the row effects but were substantially less powerful for detecting the column and interaction effects.

2 . All the approaches examined were substantially affected by the relationship between the patterns of cell variances and cell sizes. In particular, when our interest was focused on comparing the sensitivity of the procedures for detecting the row effects, the power rates were much larger under the condition of positive pairings than under the condition of negative pairings, especially when $C=$ .50 . This same pattern of results was obtained with the original BDM test and its corresponding transformations for detecting the column and interaction effects. However, the same did not occur with the standard WJ test and its corresponding transformations. In this case, the results in Table 5 indicate that the empirical power rates were much larger when variances and cell sizes were negatively paired.

3. Finally, Table 5 reveals that the pattern of power differences was moderately affected by the form of distribution. In general, the empirical power rates decreased when the distribution was symmetric leptokurtic. The results also show that when data were generated from an asymmetric distribution with a moderate degree of kurtosis, 
Table 4

Empirical Type I Error Rates (in Percentages) for the $3 \times 5$ Factorial Design $(C=.50)$

\begin{tabular}{|c|c|c|c|c|c|c|c|c|c|c|c|c|c|}
\hline \multirow[b]{2}{*}{$N$} & \multirow[b]{2}{*}{$\mathrm{R}(N / V)$} & \multicolumn{3}{|c|}{$\begin{array}{c}\text { Distribution } \\
g=.000, h=.000\end{array}$} & \multicolumn{3}{|c|}{$\begin{array}{c}\text { Distribution } \\
g=.000, h=.109\end{array}$} & \multicolumn{3}{|c|}{$\begin{array}{c}\text { Distribution } \\
g=.76, h=-.098\end{array}$} & \multicolumn{3}{|c|}{$\begin{array}{c}\text { Distribution } \\
g=1.00, h=.000\end{array}$} \\
\hline & & $\mathrm{H}_{\mathrm{R}}$ & $\mathrm{H}_{\mathrm{C}}$ & $\mathrm{H}_{\mathrm{I}}$ & $\mathrm{H}_{\mathrm{R}}$ & $\mathrm{H}_{\mathrm{C}}$ & $\mathrm{H}_{\mathrm{I}}$ & $\mathrm{H}_{\mathrm{R}}$ & $\mathrm{H}_{\mathrm{C}}$ & $\mathrm{H}_{\mathrm{I}}$ & $\mathrm{H}_{\mathrm{R}}$ & $\mathrm{H}_{\mathrm{C}}$ & $\mathrm{H}_{\mathrm{I}}$ \\
\hline \multicolumn{14}{|c|}{ S-BDM } \\
\hline 104 & + & 4.83 & 4.67 & 4.22 & 4.33 & 4.48 & 4.34 & 4.09 & 3.04 & 2.36 & 3.18 & 1.73 & 1.41 \\
\hline 104 & - & 5.02 & 4.70 & 4.83 & 4.08 & 4.10 & 3.67 & 4.17 & 4.61 & 2.68 & 3.47 & 4.50 & 1.62 \\
\hline 208 & + & 4.81 & 4.59 & 4.62 & 4.65 & 4.36 & 4.77 & 4.62 & 4.39 & 4.05 & 4.37 & 3.01 & 1.84 \\
\hline 208 & - & 5.25 & 5.01 & 5.02 & 4.79 & 4.61 & 4.56 & 5.06 & 4.69 & 4.32 & 4.83 & 4.61 & 1.99 \\
\hline \multicolumn{14}{|c|}{$\mathrm{H}-\mathrm{BDM}$} \\
\hline 104 & + & 5.54 & 5.54 & 5.83 & 6.12 & 6.67 & 6.72 & 5.35 & 4.94 & 4.68 & 5.64 & 4.69 & 4.21 \\
\hline 104 & - & 6.10 & 6.36 & 6.42 & 5.38 & 5.52 & 5.65 & 5.60 & 6.38 & 5.19 & 5.82 & 7.57 & 4.97 \\
\hline 208 & + & 5.40 & 5.48 & 4.92 & 6.82 & 6.65 & 6.86 & 5.03 & 4.59 & 4.37 & 5.91 & 5.11 & 4.63 \\
\hline 208 & - & 5.34 & 5.70 & 5.06 & 6.18 & 6.10 & 7.08 & 4.57 & 5.14 & 3.85 & 4.98 & 5.75 & 4.09 \\
\hline \multicolumn{14}{|c|}{$\mathrm{BC}-\mathrm{BDM}$} \\
\hline 104 & + & 4.41 & 4.23 & 4.35 & 3.64 & 3.81 & 3.70 & 6.61 & 5.60 & 4.23 & 8.60 & 6.50 & 3.14 \\
\hline 104 & - & 4.59 & 4.69 & 4.71 & 4.26 & 3.08 & 3.96 & 6.50 & 6.99 & 4.54 & 7.82 & 8.82 & 3.57 \\
\hline 208 & + & 3.16 & 4.36 & 4.37 & 4.37 & 4.82 & 4.44 & 8.62 & 7.68 & 5.16 & 13.67 & 13.27 & 4.86 \\
\hline 208 & - & 4.11 & 4.62 & 4.79 & 4.06 & 4.53 & 4.68 & 7.94 & 8.31 & 5.60 & 11.02 & 12.20 & 4.58 \\
\hline \multicolumn{14}{|l|}{ S-WJ } \\
\hline 104 & + & 5.23 & 5.27 & 4.49 & 4.30 & 3.90 & 3.52 & 4.58 & 4.73 & 3.91 & 4.16 & 4.92 & 3.02 \\
\hline 104 & - & 4.68 & 4.76 & 5.25 & 3.52 & 4.20 & 4.24 & 4.49 & 9.78 & 8.10 & 4.73 & 14.42 & 7.65 \\
\hline 208 & + & 4.74 & 4.92 & 5.22 & 4.46 & 4.46 & 4.50 & 4.92 & 5.46 & 4.49 & 4.98 & 5.93 & 3.70 \\
\hline 108 & - & 5.19 & 5.31 & 4.74 & 4.90 & 5.06 & 4.45 & 4.97 & 8.57 & 6.38 & 5.25 & 13.99 & 6.82 \\
\hline \multicolumn{14}{|l|}{$\mathrm{H}-\mathrm{WJ}$} \\
\hline 104 & + & 5.97 & 6.28 & 7.09 & 6.04 & 6.50 & 7.40 & 5.22 & 5.82 & 6.34 & 6.56 & 7.53 & 8.05 \\
\hline 104 & - & 5.72 & 5.87 & 7.41 & 5.45 & 5.82 & 7.88 & 5.80 & 10.01 & 9.74 & 6.56 & 15.11 & 12.06 \\
\hline 208 & + & 5.29 & 5.40 & 6.04 & 6.04 & 6.52 & 7.42 & 5.19 & 5.07 & 3.61 & 6.53 & 6.31 & 3.86 \\
\hline 208 & - & 5.59 & 5.58 & 5.34 & 6.30 & 6.08 & 7.76 & 4.56 & 6.52 & 4.28 & 5.49 & 11.35 & 5.16 \\
\hline \multicolumn{14}{|c|}{$\mathrm{BC}-\mathrm{WJ}$} \\
\hline 104 & + & 4.61 & 4.65 & 4.51 & 3.66 & 3.76 & 3.40 & 6.29 & 5.78 & 4.63 & 8.67 & 7.62 & 4.42 \\
\hline 104 & - & 4.06 & 4.70 & 5.30 & 3.08 & 4.16 & 4.31 & 6.12 & 10.97 & 9.17 & 7.71 & 17.07 & 9.23 \\
\hline 208 & + & 4.02 & 4.42 & 5.05 & 3.64 & 3.92 & 4.18 & 8.91 & 7.91 & 5.24 & 14.53 & 12.64 & 5.65 \\
\hline 208 & - & 4.23 & 4.86 & 4.54 & 4.20 & 4.54 & 4.36 & 7.85 & 11.85 & 7.15 & 11.35 & 20.79 & 8.48 \\
\hline
\end{tabular}

Note- $g=(\cdot), h=(\cdot)$ specifies a particular $g$-and- $h$ distribution with specific values of skewness and kurtosis; $C$, coefficient of cell size variation; $N$, total sample size; $\mathrm{R}(N / V)$, relationship between the size of the cells and the size of the variances; $\mathrm{H}_{\mathrm{R}}$, hypothesis rows; $\mathrm{H}_{\mathrm{C}}$, hypothesis columns; $\mathrm{H}_{\mathrm{I}}$, hypothesis interaction; $\mathrm{S}-\mathrm{BDM}$, standard Brunner-Dette-Munk test; H-BDM, Hall's transformation BDM test; BC-BDM, BDM test based on Box-Cox's power transformation; S-WJ, standard Welch-James test; H-WJ, Hall's transformation WJ test; $\mathrm{BC}-\mathrm{WJ}$, WJ test based on Box-Cox's power transformation. Bold values are outside the $[2.50-7.50]$ interval, for the $5 \%$ level of significance used in this study.

the heteroscedastic statistics based on Hall's (1992) transformation were slightly more powerful than the original $\mathrm{BDM}$ and $\mathrm{WJ}$ tests and their corresponding versions based on Box-Cox's transformation of the data. However, it can be seen that the power rates of the standard BDM and WJ tests were scarcely affected by the transformations when the distribution was normal.

\section{Discussion and Recommendations}

Previous studies have shown that using Hall's (1992) transformation with trimmed means improves the performance of Johansen's (1980) test when the assumptions of homogeneity and normality are violated (Luh \& Guo, 2004). Brunner et al.'s (1997) test also limits the number of Type I errors to the alpha level; however, this method can be affected by asymmetric distributions (Vallejo et al., 2010). The present results are consistent with those obtained in the aforementioned studies. Moreover, they include new findings that aid researchers in the selection of feasible alternatives for the exploration of factorial effects.

Regarding the control of the error rates, it is noteworthy that none of the tests assessed proved to be effective under every condition manipulated. When the data were sampled from symmetric distributions, the original BDM and WJ methods were scarcely affected by the lack of homogeneity and by the lack of normality; when they were sampled from distributions with varying degrees of asymmetry, the results were inconsistent. Under the asymmetric distribution with moderate skew, Hall's (1992) transformation of the BDM test resulted in Type I error rates that remain valid with respect to Bradley's (1978) criterion, whereas the performance of the original WJ test, Hall's transformation of the $\mathrm{WJ}$ test, and the $\mathrm{WJ}$ test based on Box-Cox's data transformation resulted in liberal error rates when variances and cell sizes were negatively paired. Under the asymmetric distribution with severe skew, no approach was capable of controlling the error rates. The original BDM test resulted in excessively conservative rates, whereas the remaining procedures studied tended to yield liberal values. However, the degree of liberalness of the tests based on Hall's transformation decreased as total sample size increased. It is also important to note that the results revealed that the tests based on Hall's transformation method substantially outperformed those based on Box-Cox's transformation when the data were sampled from skewed distributions. In contrast, the tests based on 
Table 5

Empirical Power Rates (in Percentages) for the $3 \times 5$ Factorial Designs (Target $=.60$ )

\begin{tabular}{|c|c|c|c|c|c|c|c|c|c|c|c|c|c|}
\hline \multirow[b]{3}{*}{$N$} & \multirow[b]{3}{*}{$\mathrm{R}(N / V)$} & \multicolumn{6}{|c|}{$C=.25$} & \multicolumn{6}{|c|}{$C=.50$} \\
\hline & & \multicolumn{3}{|c|}{$\begin{array}{c}\text { Distribution } \\
g=.000, h=.000\end{array}$} & \multicolumn{3}{|c|}{$\begin{array}{c}\text { Distribution } \\
g=.000, h=.109\end{array}$} & \multicolumn{3}{|c|}{$\begin{array}{c}\text { Distribution } \\
g=.000, h=.000\end{array}$} & \multicolumn{3}{|c|}{$\begin{array}{c}\text { Distribution } \\
g=.000, h=.109\end{array}$} \\
\hline & & $\mathrm{H}_{\mathrm{R}}$ & $\mathrm{H}_{\mathrm{C}}$ & $\mathrm{H}_{\mathrm{I}}$ & $\mathrm{H}_{\mathrm{R}}$ & $\mathrm{H}_{\mathrm{C}}$ & $\mathrm{H}_{\mathrm{I}}$ & $\mathrm{H}_{\mathrm{R}}$ & $\mathrm{H}_{\mathrm{C}}$ & $\mathrm{H}_{\mathrm{I}}$ & $\mathrm{H}_{\mathrm{R}}$ & $\mathrm{H}_{\mathrm{C}}$ & $\mathrm{H}_{\mathrm{I}}$ \\
\hline \multicolumn{14}{|c|}{ S-BDM } \\
\hline 104 & + & 68.1 & 60.4 & 54.4 & 53.6 & 42.8 & 36.6 & 71.3 & 65.3 & 59.6 & 55.2 & 46.9 & 38.0 \\
\hline 104 & - & 56.6 & 43.2 & 37.9 & 44.4 & 30.4 & 25.9 & 34.6 & 23.4 & 19.3 & 26.6 & 17.8 & 14.4 \\
\hline 208 & + & 64.4 & 62.0 & 56.5 & 83.3 & 42.9 & 39.8 & 65.8 & 68.9 & 63.4 & 49.6 & 49.8 & 43.6 \\
\hline \multirow[t]{2}{*}{208} & - & 58.5 & 46.5 & 41.8 & 73.9 & 31.8 & 29.9 & 35.9 & 25.4 & 21.6 & 27.9 & 20.2 & 16.9 \\
\hline & & 61.9 & 53.0 & 47.7 & 63.8 & 37.0 & 33.1 & 51.9 & 45.8 & 41.0 & 39.8 & 33.7 & 28.2 \\
\hline \multicolumn{14}{|c|}{ H-BDM } \\
\hline 104 & + & 69.7 & 61.7 & 57.7 & 58.1 & 49.0 & 46.8 & 71.7 & 65.9 & 60.8 & 59.5 & 53.9 & 49.2 \\
\hline 104 & - & 58.5 & 44.1 & 41.3 & 49.0 & 38.4 & 35.4 & 36.6 & 24.4 & 21.7 & 31.1 & 21.7 & 18.3 \\
\hline 208 & + & 63.5 & 63.0 & 57.0 & 83.9 & 49.9 & 48.2 & 66.6 & 68.5 & 63.5 & 54.9 & 56.4 & 52.5 \\
\hline \multirow[t]{2}{*}{208} & - & 57.9 & 47.0 & 43.5 & 76.9 & 38.2 & 37.7 & 36.9 & 26.9 & 22.3 & 31.9 & 25.9 & 23.2 \\
\hline & & 62.4 & 53.4 & 49.9 & 66.9 & 43.9 & 42.0 & 52.9 & 46.4 & 42.1 & 44.4 & 39.5 & 35.8 \\
\hline \multicolumn{14}{|c|}{ BC-BDM } \\
\hline 104 & + & 71.6 & 50.9 & 51.6 & 57.8 & 34.7 & 34.6 & 76.2 & 46.6 & 47.9 & 61.2 & 31.1 & 32.9 \\
\hline 104 & - & 60.3 & 54.6 & 46.5 & 46.4 & 38.2 & 31.9 & 37.6 & 37.2 & 34.6 & 29.1 & 25.8 & 24.4 \\
\hline 208 & + & 67.6 & 50.4 & 53.0 & 86.8 & 32.0 & 38.6 & 72.8 & 46.8 & 50.8 & 57.8 & 30.9 & 34.8 \\
\hline \multirow[t]{2}{*}{208} & - & 58.9 & 61.4 & 50.2 & 78.9 & 43.3 & 33.4 & 39.9 & 43.5 & 37.1 & 31.9 & 32.6 & 26.1 \\
\hline & & 64.6 & 54.3 & $\mathbf{5 0 . 3}$ & 67.5 & 37.0 & 34.6 & 56.5 & 43.5 & 42.6 & 45.0 & 30.1 & 29.6 \\
\hline \multicolumn{14}{|l|}{ S-WJ } \\
\hline 104 & + & 63.3 & 75.0 & 72.9 & 49.7 & 59.8 & 52.7 & 66.7 & 61.6 & 56.8 & 52.9 & 47.7 & 43.0 \\
\hline 104 & - & 51.1 & 88.8 & 73.5 & 40.5 & 72.3 & 58.1 & 31.3 & 80.1 & 65.2 & 24.6 & 63.3 & 48.4 \\
\hline 208 & + & 57.1 & 78.5 & 76.8 & 80.9 & 62.9 & 60.3 & 61.0 & 67.8 & 64.4 & 46.5 & 51.9 & 49.1 \\
\hline \multirow[t]{2}{*}{108} & - & 48.9 & 91.4 & 79.4 & 71.5 & 74.6 & 62.2 & 34.7 & 87.1 & 77.3 & 26.9 & 71.6 & 60.1 \\
\hline & & 55.1 & 83.4 & 75.6 & 60.7 & 67.4 & 58.3 & 48.4 & 74.2 & 65.9 & 37.7 & 58.6 & 50.2 \\
\hline \multicolumn{14}{|l|}{ H-WJ } \\
\hline 104 & + & 65.6 & 73.7 & 70.0 & 55.8 & 62.8 & 63.7 & 67.4 & 61.7 & 57.6 & 57.4 & 50.0 & 51.5 \\
\hline 104 & - & 53.2 & 86.4 & 71.1 & 46.3 & 73.6 & 64.8 & 33.2 & 78.4 & 65.7 & 29.1 & 66.3 & 56.4 \\
\hline 208 & + & 59.1 & 76.5 & 75.0 & 81.4 & 65.3 & 66.5 & 62.6 & 67.8 & 63.6 & 51.6 & 55.8 & 56.7 \\
\hline \multirow[t]{2}{*}{208} & - & 50.3 & 90.1 & 78.6 & 73.5 & 77.4 & 68.4 & 35.5 & 87.8 & 76.7 & 31.4 & 74.1 & 65.6 \\
\hline & & 57.1 & 81.7 & 73.7 & 64.3 & 69.8 & 65.9 & 49.7 & 73.9 & 65.9 & 42.4 & 61.6 & $\mathbf{5 7 . 5}$ \\
\hline \multicolumn{14}{|c|}{ BC-WJ } \\
\hline 104 & + & 65.8 & 73.6 & 72.4 & 50.3 & 56.7 & 56.6 & 68.1 & 57.0 & 57.1 & 53.9 & 43.3 & 44.2 \\
\hline 104 & - & 52.3 & 90.9 & 72.0 & 40.7 & 73.6 & 58.4 & 31.5 & 81.5 & 65.7 & 23.5 & 64.4 & 48.8 \\
\hline 208 & + & 60.0 & 74.1 & 77.9 & 82.2 & 56.0 & 60.2 & 64.8 & 56.9 & 65.7 & 50.7 & 44.9 & 50.7 \\
\hline \multirow[t]{2}{*}{208} & - & 50.7 & 93.2 & 78.1 & 72.7 & 73.8 & 61.3 & 34.2 & 89.9 & 75.7 & 26.3 & 72.0 & 59.7 \\
\hline & & 57.2 & 82.9 & 75.1 & 61.5 & 64.8 & 59.1 & 49.7 & 71.3 & 66.0 & 38.6 & 56.2 & 50.9 \\
\hline
\end{tabular}

Note $-g=(\cdot), h=(\cdot)$ specifies a particular $g$-and- $h$ distribution with specific values of skewness and kurtosis; $C$, coefficient of cell size variation; $N$, total sample size; $\mathrm{R}(N / V)$, relationship between the size of the cells and the size of the variances; $\mathrm{H}_{\mathrm{R}}$, hypothesis rows; $\mathrm{H}_{\mathrm{C}}$, hypothesis columns; $\mathrm{H}_{\mathrm{I}}$, hypothesis interaction; $\mathrm{S}-\mathrm{BDM}$, standard Brunner-Dette-Munk test; H-BDM, Hall's transformation BDM test; BC-BDM, BDM test based on Box-Cox's power transformation; S-WJ, standard Welch-James test; H-WJ, Hall's transformation WJ test; BC-WJ, WJ test based on Box-Cox's power transformation. Boldface entries represent the average of the quantities above.

Box-Cox's transformation slightly outperformed those based on Hall's transformation when the data were sampled from a symmetric distribution with a moderate degree of kurtosis, particularly for Hall's transformation of the WJ test. On the whole, the results revealed that Hall's transformation of the BDM test was consistently more robust than Hall's transformation of the WJ test for most of the conditions studied. Accordingly, Hall's transformation of the BDM test appears to be the best choice. These results are consistent with those reported by several authors, including Luh and Guo (2004) and Vallejo et al. (2010). They also serve to highlight the importance of verifying whether the data presented a deviation from normality prior to their analysis.

Consistent with the results of previous research, we found that no procedure was uniformly more powerful than the others. In general, both the original BDM and the transformed BDM methods were slightly more powerful than the original WJ and modified WJ methods for detecting the row effects, but substantially less powerful for detecting the column and the interaction effects, respectively. This large difference in power was observed for both moderate and severe degrees of cell size imbalance, regardless of the population distribution shape. In addition, when the distribution was normal, the power rates of the original BDM and WJ tests were scarcely affected by the transformations. Specifically, for original and transformed BDM methods, the average power values were $50 \%$ and $51 \%$, respectively, whereas the corresponding WJ values were $67 \%$ and $67 \%$. However, the results also show that when data were generated from a symmetric distribution with a moderate degree of kurtosis, the heteroscedastic statistics based on Hall's (1992) transformation were slightly more powerful than the original BDM 
and $\mathrm{WJ}$ tests and their corresponding versions based on the transformation of data using Box-Cox's power method. Once more, these results confirm and generalize those found in similar studies (Luh \& Guo, 2004; Vallejo et al., 2010). It is also important to note that the conclusions regarding the sensitivity of these procedures are based only on one type of mean configuration; further investigation involving some other types of population mean configurations may well lead to a modification of the suggestions made here.

Thus, in order to apply the investigated methods appropriately, the results of our study revealed that researchers should ideally be familiar with the distributional shape of populations. Although the choice of a procedure for statistical inference in heteroscedastic factorial designs in the presence of nonnormal data is complex, a preliminary formal test for symmetry can be used to test whether the distributions are symmetric or not. Othman, Keselman, Wilcox, Padmanabhan, and Fradette (2002) provided an SAS/ IML program to compute a test of symmetry, based on the work of Hogg, Fisher, and Randles (1975) and Babu, Padmanabhan, and Puri (1999). It is important to note, however, that this test is asymptotically valid and, therefore, cannot be reliable for small sample sizes. Graphical methods (e.g., boxplots and variations, all available in SAS and SPSS) can also be examined for evidence of skewness in the data. If the distributions are found to be symmetric, on the basis of our results, we recommend using either of the WJ procedures, since they were generally more powerful than their most direct competitors and, in most conditions, substantially more powerful. Keselman, Wilcox, and Lix (2003) presented an SAS/IML program for implementing the $\mathrm{WJ}$ test for any factorial design. Otherwise, for moderate degrees of skewness (e.g., exponential-type data), we consider using Hall's transformation of the BDM statistic to be more justified.

To implement Hall's (1992) transformation of the BDM statistic described in the Definition of the Statistical Tests section, we developed a computational program (available from the first author) written in the SAS/IML programming language. The program calculates the BDM solution for tests of the main and interaction effects in factorial designs. In addition, contrasts among unweighted marginal means or all possible interaction contrasts (i.e., tetrad contrasts) can be obtained by applying Hochberg's (1988) sequentially rejective Bonferroni procedure. All of the $F$ tests and the Hochberg adjusted $p$ values are calculated automatically by the program.

To conclude, we would like to add four brief comments. First, the results are limited to the conditions examined in the study, although we sense that they can be generalized to a wider range of conditions. Second, when the shape of the distribution is severely skewed (e.g., lognormaltype data), choosing a completely valid alternative is complex, although the deleterious effects of nonnormality and heterogeneity can also generally be overcome using the original BDM approach based on Hall's (1992) transformation method. Another option for handling nonnormal data directly is to use the bootstrap version of Hall's transformation (Zhou \& Gao, 2000) and permutation resam- pling methods. However, for a very small sample size, the bootstrap resampling methods (e.g., percentile- $t$ bootstrap, Hall's bootstrap) should be used with caution (Luh \& Guo, 2004). For this reason, in future research, it would also be informative to examine the performance of the linear model, using techniques that allow distributions of error terms other than the normal, and relax the requirement of constant variability. These techniques, known as generalized linear models, are an extension of the general linear model in which the distribution of the error terms is not restricted to being normal but is widened to accommodate many of the most important distributions encountered when we work with real data, including all those examined here. More detailed information can be found in McCullagh and Nelder (1989), Dobson and Barnett (2008), McCulloch, Searle, and Neuhaus (2008), and the references cited therein. Model estimation and inference for the fixed effects can be easily performed with standard statistical packages, such as SAS (fit by the GLIMMIX procedure) and SPSS (fit by the GENLIN command). PROC GLIMMIX allows fitting statistical models to data when assumptions of normality and variance homogeneity are not necessarily satisfied (see Schabenberger, 2005, 2007, for details and suggestions; see also Kenward \& Roger, 2009, for warnings with the Kenward-Roger degrees-of-freedom correction). However, choices as to the form of distribution and link function still have to be made. Several approximations have been suggested to overcome the problem (e.g., Cerrito, 2005; Czado \& Raftery, 2006). Third, the analyses were conducted only for two-factor designs with fixed levels, and these approaches can also be applied in the analysis of higher factorial designs containing one or more dependent variables. An SAS macro that performs the BDM approach for a variety of univariate and multivariate designs is available from the first author.

Finally, one caveat is included. The choice of original scale of measurement is often quite arbitrary, so that a change of scale may be preferable if the distribution on the new scale has desirable properties (e.g., normality, homoscedasticity). Under such circumstances, we consider that the transformations are a viable solution. However, it may happen that as a result of a monotone (i.e., consistently preserves the ordering of the cell means but does not preserve the relative distances among them) transformation of the data, not only the skewness of the parent distributions can be modified, but also some of the factorial effects defined in the linear model. For example, if means for the levels of variable $A$ have the same rank for all levels of variable $B$, a transformation can potentially remove the $A B$ interaction (see Quinn \& Keough, 2002).

\section{AUTHOR NOTE}

This work was supported by Grant PSI2008-03624 from the Spanish Ministry of Science and Innovation and by Grant PSI2005-09002 from the Spanish Ministry of Education and Science. We gratefully thank the anonymous reviewers and the associate editor for their helpful comments and very constructive suggestions. Correspondence concerning this article should be addressed to G. Vallejo, Department of Psychology, University of Oviedo, Plaza de Benito Feijóo, s/n, 33003 Oviedo, Spain (e-mail: gvallejo@uniovi.es). 


\section{REFERENCES}

AKRITAS, M. G. (1990). The rank transform method in some two-factor designs. Journal of the American Statistical Association, 85, 73-78.

Babu, J. G., Padmanabhan, A. R., \& Puri, M. P. (1999). Robust oneway ANOVA under possibly non-regular conditions. Biometrical Journal, 41, 321-339.

Box, G. E. P. (1954). Some theorems on quadratic forms applied in the study of analysis of variance problems: I. Effects of inequality of variance in the one-way classification. Annals of Mathematical Statistics, 25, 290-403.

Box, G. E. P., \& Cox, D. R. (1964). An analysis of transformations. Journal of the Royal Statistical Society B, 26, 211-246.

Bradley, J. (1978). Robustness? British Journal of Mathematical \& Statistical Psychology, 31, 144-152.

Brunner, E., Dette, H., \& Munk, A. (1997). Box-type approximations in heteroscedastic factorial designs. Journal of the American Statistical Association, 92, 1494-1503.

Cerrito, P. B. (2005, October). From GLM to GLIMMIX-Which model to choose? Paper presented at the 13th Annual Conference of the Southeast SAS Users Group, Portsmouth, VA.

Czado, C., \& Raftery, A. E. (2006). Choosing the link function and accounting for link uncertainty in generalized linear models using Bayes factors. Statistical Papers, 47, 419-442.

Dobson, A. J., \& BARNETT, A. G. (2008). Introduction to generalized linear models (3rd ed.). London: Chapman \& Hall/CRC.

HALL, P. (1992). On the removal of skewness by transformation. Journal of the Royal Statistical Society: Series B, 54, 221-228.

Headrick, T. C., Kowalchuk, R. K., \& Sheng, Y. (2008). Parametric probability densities and distribution functions for Tukey $g$-and- $h$ transformations and their use for fitting data. Applied Mathematical Sciences, 2, 449-462.

Hoaglin, D. C. (1985). Summarizing shape numerically: The g-and-h distributions. In D. C. Hoaglin, F. Mosteller, \& J. W. Tukey (Eds.), Exploring data tables, trends, and shapes (pp. 461-513). New York: Wiley.

HochBERG, Y. (1988). A sharper Bonferroni procedure for multiple tests of significance. Biometrika, 75, 800-802.

HogG, R. V., Fisher, D. M., \& Randles, R. H. (1975). A two-sample adaptive distribution free test. Journal of the American Statistical Association, 70, 656-661.

JAMES, G. S. (1951). The comparison of several groups of observations when the ratios of the population variances are unknown. Biometrika, 38, 324-329.

Johansen, S. (1980). The Welch-James approximation to the distribution of the residual sum of squares in a weighted linear regression. Biometrika, 67, 85-92.

JoHnson, N. J. (1978). Modified $t$ tests and confidence intervals for asymmetrical populations. Journal of the American Statistical Association, 92, 85-92.

Kenward, M. G., \& Roger, J. H. (2009). An improved approximation to the precision of fixed effects from restricted maximum likelihood. Computational Statistics \& Data Analysis, 53, 2583-2595.

Keselman, H. J., Carriere, K. C., \& Lix, L. M. (1995). Robust and powerful nonorthogonal analyses. Psychometrika, 60, 395-418.

Keselman, H. J., KowalchuK, R. K., \& Lix, L. M. (1998). Robust nonorthogonal analyses revisited: An update based on trimmed means. Psychometrika, 63, 145-163.

Keselman, H. J., Wilcox, R. R., \& Lix, L. M. (2003). A generally robust approach to hypothesis testing in independent and correlated groups designs. Psychophysiology, 40, 586-596.
Krishnamoorthy, K., Lu, F., \& Mathew, T. (2007). A parametric bootstrap approach for ANOVA with unequal variances: Fixed and random models. Computational Statistical \& Data Analysis, 51, 57315742.

Kulinskaya, E., \& Dollinger, M. B. (2007). Robust weighted oneway ANOVA: Improved approximation and efficiency. Journal of Statistical Planning \& Inference, 137, 462-472.

LEe, S., \& AHN, C. H. (2003). Modified ANOVA for unequal variances. Communications in Statistics: Simulation \& Computation, 32, 9871004.

LuH, W. M., \& GuO, J. H. (2004). Improved robust test statistic based on trimmed means and Hall's transformation for two-way ANOVA models under non-normality. Journal of Applied Statistics, 31, 623-643.

MacNaughton, D. B. (1998). Which sums of squares are best in unbalanced analysis of variance? Available at www.matstat.com/ss.

Martinez, J., \& Iglewicz, B. (1984). Some properties of the Tukey g-and-h family of distributions. Communications in Statistics: Theory \& Methods, 13, 353-369.

Maxwell, B. E., \& Delaney, H. D. (2004). Designing experiments and analyzing data: A model comparison perspective. Mahwah, $\mathrm{NJ}$ : Erlbaum.

McCullagh, P., \& Nelder, J. (1989). Generalized linear models (2nd ed.). London: Chapman \& Hall/CRC.

McCulloch, C. E., Searle, S. R., \& Neuhaus, J. W. (2008). Generalized, linear, and mixed models (2nd ed.). New York: Wiley.

MiCCERI, T. (1989). The unicorn, the normal curve, and other improbable creatures. Psychological Bulletin, 92, 778-785.

Othman, A. R., Keselman, H. J., Wilcox, R. R., Padmanabhan, A. R. \& FradeTte, K. (2002). A description and illustration of a test of symmetry. Journal of Modern Applied Statistical Methods, 2, 310-315.

Quinn, G. P., \& Keough, M. J. (2002). Experimental design and data analysis for biologists. Cambridge: Cambridge University Press.

Richter, S. J., \& PAYTON, M. E. (2003). Performing two-way analysis of variance. Journal of Modern Applied Statistical Methods, 2, 152-160.

SAS Institute Inc. (2008). SAS/STAT ${ }^{\circledR} 9.2$ user's guide. Cary, NC: Author.

Schabenberger, O. (2005). Introducing the GLIMMIX procedure for generalized linear mixed models. In Proceedings of the Thirtieth Annual SAS Users Group International Conference (Paper 196-30). Cary, NC: SAS Institute.

SCHABENBERGER, O. (2007). Growing Up Fast: SAS ${ }^{\circledR} 9.2$ Enhancements to the GLIMMIX procedure. In Proceedings of the 2007 SAS Global Forum (pp. 1-20). Cary, NC: SAS Institute.

Tukey, J. W. (1977). Modern techniques in data analysis. Paper presented at the NSF-sponsored regional research conference at Southeastern Massachusetts University, North Dartmouth.

Vallejo, G., \& Aто, M. (2006). Modified Brown-Forsythe procedure for testing interaction effects in split-plot designs. Multivariate Behavioral Research, 41, 549-578.

Vallejo, G., Fernández, M. P., \& Livacic-Rojas, P. E. (2010). Analysis of unbalanced factorial designs with heteroscedastic data. Journal of Statistical Computation \& Simulation, 80, 75-88. doi:10.1080/ 00949650802482386

WeLCH, B. L. (1951). On the comparison of several mean values: An alternative approach. Biometrika, 38, 330-336.

Zhou, X. H., \& GAO, S. (2000). One-sided confidence intervals for means of positively skewed distributions. American Statistician, 54, 100-104.

(Manuscript received June 17, 2009; revision accepted for publication November 13, 2009.) 